

\title{
9 conceptualización de pobreza desde la perspectiva de género
}

\author{
M arcelina Castillo Venerio
}

\section{INTRODUCCIÓN}

Este artículo es un fragmento de un estudio realizado para el Instituto N icaragüense de la M ujer (IN IM ) con el apoyo del Programa de $\mathrm{N}$ aciones $\mathrm{U}$ nidas para el Desarrollo (PNUD), en el que se pretende esbozar un marco conceptual para el enfoque y pautas para la construcción de los indicadores referentes a la Pobreza y la Economía desde la Perspectiva de $\mathrm{G}$ énero que sirva de referencia a la implementación del enfoque de género en el sistema de estadísticas nacionales. Con ello se pretende favorecer la equidad entre mujeres y hombres como un requisito para lograr el desarrollo pleno.

Contiene tres apartados. En el primero se presenta cómo ha evolucionado el concepto de la pobreza en los últimos cincuenta años; en el segundo, nuevos conceptos asociados a la pobreza y el tercero, los métodos de medición de la pobreza.

\section{EVOLUCIÓN DEL CONCEPTO DE POBREZA}

A la fecha no existe un consenso sobre la conceptualización y formas de medición de la pobreza. El cuerpo teórico del concepto de pobreza es muy disperso y está constituido por enfoques conceptuales diferentes que se reducen a dos: el enfoque de la pobreza absoluta y el enfoque estructural de la pobreza.

\subsection{Evolución del concepto de Pobreza}

El estudio de la pobreza se remonta a finales del siglo XIX y comienzos del siglo XX. A Igunas estimaciones sobre pobreza se realizan a finales del siglo XIX en Londres. El primero en combinar la observación con un intento sistemático de medición de la extensión del problema de la pobreza fue Booth, quien elaboró un mapa de pobreza de Londres entre 1892 y 1897. En 1901, Rowtree realizó un estudio para medir la pobreza en Cork, utilizando un estándar de pobreza basado en requerimientos nutricionales (A tkinson: 1987).

A partir del siglo XX se han desarrollado nuevos conceptos y metodologías sobre la medición de la pobreza. A ntes de los años setenta, la pobreza se explicaba en base a aspectos monetarios. Para definir la condición de pobreza se usaba el ingreso por persona o el ingreso por familia, estableciéndose un ingreso por persona de un dólar diario. De ahí surgió el término de "línea de pobreza" definiéndose a partir del ingreso de las familias en relación al costo de la canasta básica en el país. Estas formas de explicar la pobreza son consideradas insuficientes.

A partir de 1973 se dan inicio los debates sobre cuál es la mejor forma de definir y medir la pobreza. Es en la década de los setenta que se empieza a desarrollar una nueva conceptualización de la pobreza. En ese mismo período también se debate sobre el rol del enfoque de género en el desarrollo, en las políticas económicas y en el análisis de la pobreza. En este contexto se dieron fuertes críticas a la idea de 'neutralidad' de las políticas macroeconómicas y a la presentación del hogar como una unidad (v. Punto........macroeconomía).

En los ochenta el enfoque aún se basaba en aspectos no monetarios, lo que hizo más complejo el debate, particularmente con la inclusión de algunos términos como indefención (powerlesness), capacidades (capabilities) formas sostenibles de vivir (sustainable livilihoods). ${ }^{1}$ En esta misma década, la pobreza se define como una forma de exclusión del sistema moderno de producción y una inserción en los sistemas precapitalistas de producción. Este enfoque habla más de pobres que de pobreza y está interesado en la cultura de la pobreza y en las estrategias de los pobres. La pobreza fue definida como una fal ta de consumo de bienes básicos. El pobre aparece como parte de un problema coyuntural de infraconsumo. También se consideró como una combinación de al gunas carencias básicas y como un proceso de desacumulación en el cual los activos del hogar van disminuyendo. (CEPA L, 2002). Esta definición también resulta limitada porque es totalmente economicista, puesto que la pobreza es un fenómeno más complejo que comprende no sólo la insuficiencia de recursos monetarios sino también otras privaciones y exclusiones.

En losaños noventa losimpactos negativos sobre la pobreza de las políticas de ajuste estructural motivaron la discusión de nuevos conceptos como "vulnerabilidad", basado en una gama más amplia de elementos, como el de "exclusión social", acuñado por los franceses (M oser; 1996). A partir de estos nuevos conceptos se considera que una persona es pobre cuan do se encuentra en situación de privación, de exclusión de los procesos productivos, cuando no está integrada socialmente y/o carece de acceso de oportunidades. En este sentido, para que un hogar pueda satiffacer las necesidades básicas de sus integrantes, Julio Boltvinik ${ }^{2}$ ubica cinco variables que determinan tal satisfacción: consumo como indicador de acceso; derechos de acceso a servicios gubernamental es; propiedad o derecho de 
uso de activos; tiempo disponible que incluye un ingreso adicional para pago de servicios domésticos y; desahorro.

La CEPA L y otras agencias del Sistema de $\mathrm{N}$ aciones $\mathrm{U}$ nidas han avanzado en la conceptualización de la problemática de la calidad de vida y pobreza. La definición de pobreza que elabora el Programa de las $\mathrm{N}$ aciones U nidas para el D esarrollo (PNUD), conceptualiza a la pobreza como ausencia de satisfactores apropiados para cubrir un mínimo de ciertas necesidades Ilamadas básicas, las cuales requieren de un esfuerzo productivo para su satisfacción. Las necesidades son: alimentación, cuidado de la salud, uso de vivienda, educación básica, vestido y calzado, transporte y comunicaciones básicas.

Toda esta evolución del concepto de la pobreza no se desliga de los enfoques, de ahí la importancia de analizarlos. A continuación se presenta una revisión de los enfoques de la pobreza más importantes.

\subsection{Dos grandes enfoques de la pobreza: pobreza absoluta y pobreza estructural}

La pobreza ha sido analizada bajo dos enfoques principales: el de la pobreza absoluta o globalizante y el de la pobreza estructural.

El enfoque de la pobreza absoluta globaliza la pobreza sin distinguir las diferencias estructurales a partir del enfoque de género. A este enfoque se atribuye la identificación de los pobrespor el monto del ingreso familiar obtenido en un período considerado. Este enfoque ha sido utilizado por la CEPA L y el Banco M undial para medir la pobreza en distintos países de A mérica Latina y la limitación que presenta es que no permite conocer aspectos de la realidad social de las personas que permitirían distinguir la situación de pobreza de las mujeres por aspectos de género.

El enfoque estructural de la pobreza es más moderno y está todavía en fase de construcción. Parte de las relaciones social mente construidas entre hombres y mujeres, es decir del género; y considera que debe tomarse en cuenta el impacto no fortuito de las causas de la pobreza, como los sesgos de género, sean cuál es fueran los contextos específicos locales, regional es y nacionales ( $v$. J anine A nderson: "Femenización de la pobreza"). Por consiguiente, analizar la situación de pobreza de las mujeres en relación a los hombres, plantea que la distinta incidencia de la pobreza en los dos géneros es el resultado de los distintos roles y posiciones de mujeres y hombres en la sociedad, derivados de la división sexual del trabajo, que asigna a los hombres el espacio público productivo y a las mujeres el espacio privado o reproductivo. Esta división sexual del trabajo por sexos incide en la desigualdad de oportunidades que tienen las personas de distinto sexo para acceder tanto a los recursos material es como a los sociales y políticos.

C abe señalar que en la medida que avanzaba la conceptualización del desarrollo tomando en consideración el enfoque de género había un avance en la construcción de un nuevo concepto de pobreza.

\subsection{Evolución de los enfoques de la pobreza desde una perspectiva de género}

Los enfoques de la pobreza, a la par de los enfoques del desarroIlo, también han evolucionado. Las características de enfoques intermedios que se dieron durante el proceso de cambio de un enfoque globalizante de la pobreza a un enfoque estructural se presentan a continuación. Para ello se utiliza la metodología planteada por Carolina M oser.

\subsubsection{El enfoque del bienestar}

El enfoque de bienestar se aplicó después de la 2da. Guerra $M$ undial a partir de identificar a las mujeres de bajos ingresos como sector vulnerable, junto con los niños pequeños, ancianos y discapacitados. Bajo esta óptica las mujeres eran receptoras pasivas de las acciones que podrían derivar en desarrollo. Cabe señalar que la unidad de análisis empleada en el enfoque de bienestar es el hogar; y prevaleció hasta los años 70.

En este enfoque, se define la pobreza como "nivel de vida", restringido este concepto al ámbito material. La identificación de los pobres implica realizar una comparación entre distintos niveles de bienestar, siendo losindicadores de bienestar más utilizados en el análisis de la pobreza el ingreso y el gasto en consumo.

Los indicadores de ingreso versus consumo planteados por el enfoque de bienestar para medir la pobreza han sido muy polémicos. $\mathrm{H}$ ay quienes consideran que la "utilidad" de las personas depende exclusivamente de su consumo presente, por consiguiente "el consumo corriente" es el indicador más apropiado del bienestar desde el punto de vista teórico, independientemente de la forma de financiamiento de ese consumo; y el ingreso es sólo una aproximación al nivel devida. ${ }^{3}$ Este planteamiento ha sido considerado como no adecuado ya que el nivel de vida de las personas no está determinado únicamente por el consumo presente, sino además por el nivel esperado de consumo futuro (A Itimir, 1979). En consecuencia, se plantea una gran cantidad de argumentos a favor y en contra de estas variables.

0 tro señalamiento que se hace es que consumo e ingreso no son suficientes por sí solos como indicadores de bienestar, sino que es necesario incluir componentes que no se observan directamente, talescomo el consumo de bienes durables y de bienes que no se transan total mente en el mercado como son: la educación y la salud. Se considera que el ingreso en especies y los servicios públicos son un factor importante en la determinación del nivel de vida, sobre todo para niveles el evados de pobreza, por consiguiente, su inclusión es necesaria (Scout, 1981). Sin embargo, hay quienes consideran que si la pobreza se define en función de la falta de recursos, no se debería incluir el ingreso en especies, puesto que las personas requieren ingresos monetarios para participar en la sociedad (A tkinson, 1991).

El mismo enfoque mide la pobreza en función del ingreso del hogar per cápita, consi derándose por al gun os como un mejor in dica- 
dor de la pobreza, ya que da cuenta del tamaño del hogar, dividiendo su ingreso total por el número de miembros. Con este enfoque el objeto de estudio de la pobreza es el hogar en vez de losindividuos lo cual permitiría analizar cómo se da la distribución del ingreso dentro de los hogares. En este sentido, el ingreso per cápita sería un mejor indicador del bienestar individual que el ingreso por hogar (Feres, 2001).

En términos dela relación pobreza y género, el enfoque de bienestar se basa en tres supuestos: que las mujeres son receptoras pasi vas del desarrollo, más que participantes activas del proceso; que la maternidad es el papel más importante de las mujeres en la sociedad; y que la crianza de los niños es el papel más efectivo en todos los aspectos del desarrollo económico (M oser, 1991: 80); y la maternidad se considera el papel principal destacado para la mujer en la sociedad y la crianza su contribución más efectiva al desarrollo. También acentúa los papeles asignados a mujeres y hombres por razones de género, correspondiendo al espacio privado y público respectivamente. $Y$ apuntaba a satisfacer las necesidades prácticas de género en el espacio reproductivo, particularmente en lo concerniente a la nutrición y la planificación familiar.

La crítica que se hace al enfoque de bienestar ha sido particularmente por usar exclusi vamente como indicadores de bienestar el consumo y el ingreso, ya que el nivel de vida no está relacionado únicamente con el aspecto material. Se ha considerado que el bienestar humano no puede medirse a partir de una sola dimensión: la económica, por consiguiente, medir el bienestar a través de un sólo indicador: el PIB per cápita esconde el problema de las relaciones entre las personas, grupos sociales y las fuerzas de poder que condicionan la distribución no sólo de los ingresos, sino también de las oportunidades que tienen las mismas para gozar de una vida digna.

D esde el feminismo, este enfoque ha sido sujeto de críticas por diferentes razones: plantea que las mujeres son el problema de la pobreza y que influyendo en su comportamiento reproductivo se podrían derivar cambios para el conjunto de la sociedad; profundiza la división de funciones con referencia al sexo; mantuvo la condición subordinada de las mujeres y profundiza su dependencia, y aumenta la carga de trabajo de lasmujeres con la modernización (Renzi, 2002).

También se considera que el interés en instrumentar este enfoque se debe a que no amenaza el status quo; es políticamente neutro y fácil de administrar.

\subsubsection{El enfoque de la equidad}

El enfoque de equidad surge en los años 70 después del reconocimiento por parte del movimiento internacional de mujeres que la contribución económica de las mujeres no se tomaba en cuenta en las estadísticas oficiales y en las cuentas nacionales.

En términos generales, el enfoque de la Equidad considera que se debe estudiar la pobreza a través del ingreso y el consumo, pero se debe corregir la variable utilizada por medio de una escal a de equiva-
Iencia que refleje de manera adecuada las necesidades económicas y materiales de cada uno de los miembros del hogar. Se basa en el concepto de escalas de equivalencia, índice que muestra el costo relativo en el que debe incurrir un hogar para gozar del mismo bienestar que un hogar de referencia, dado su tamaño y composición. Este índice agrupa dos el ementos si multán eamente. Por un lado considera las diferentes necesidades de los miembros del hogar, según edad, sexo, tipo de actividad y otras características demográficas, y por otro, el índice permite tomar en cuenta la existencia de "economías de escala", ${ }^{4}$ caracterizadas por costos marginal es decrecientes para alcanzar un mismo nivel de bienestar ante la suma de un nuevo miembro al hogar (Feres, 2001).

En término de la relación pobreza y género, el Plan de A cción $M$ undial aprobado en M éxico para la Década de la M ujer, es la referencia más importante del enfoque de equidad. ${ }^{5}$ Este enfoque reconoce que la subordinación de la mujer se da en todos los espacios de la vida pública y privada, reconocien do que la independencia económica es decisiva para el logro de la equidad; que las mujeres son activas participantes en el proceso del desarrollo, tanto en sus roles productivos como reproductivos; y que las estrategias económicas han tenido impactos negativos sobre la mujer. Revaloriza el papel crítico de las mujeres en el desarrollo con un mayor acceso al empleo y mercado.

El principal objetivo del enfoque de la equidad era reducir la desigualdad entre mujeres y hombres, especialmente en la división sexual del trabajo, para incrementar su autonomía política y económica; ya que una mayor igualdad en este plano produciría incrementos en el crecimiento económico. Debido a estas razones el enfoque se concibió por muchos como Desarrollista (R enzi, 2002).

El enfoque de equidad reconoce que las mujeres, mediante sus papeles productivos y reproductivos, son participantes activas en el proceso del desarrollo y proporcionan una contribución muy importante, a menudo no reconocida, al crecimiento económico. A pesar de ello, también ha sido sujeto de críticas. Si bien el ingreso ajustado ha sido considerado como el indicador teórico más apropiado del bienestar, el cálculo de las escalas de equivalencias ha sido controversial, dado que la forma más utilizada para construir estas escalas es la estimación de funciones de demanda a partir de la información contenida en las encuestas de gasto. 0 tro problema identificado es que la demanda potencial y efectiva no sólo depen de de las necesidades de cada miembro del hogar, sino también de la forma en que se asignan los recursos intra-familiarmentey de la construcción de identidades de género, por lo cual la escala de equivalencia podría estar replicando inequidades en la asignación de recursos dentro del hogar (Feres, 2001).

Entre las críticas que se hacen al enfoque destacan las siguientes: es reflejo de los intereses del Primer M undo; es insuficiente por la ausencia de indicadores que den cuenta del logro de la equidad; y es vertical en su imposición de arriba hacia abajo, en muchos casos promovidas por leyes o medidas legisl ativas que no responden a in- 
tereses reales de las mujeres, como lo muestra la diferencia entre la igualdad de derecho y la igualdad de hecho (Renzi, 2002).

$C$ abe señalar que este enfoque también fue rechazado muchas veces por su carácter confrontativo con el orden establecido.

\subsubsection{El enfoque antipobreza}

El enfoque antipobreza igual que los anteriores, tiene que ver con el ingreso. En el marco de este enfoque surgen nuevas formas de medición de la pobreza tales como la medición de la proporción del gasto en alimentos según el ingreso, que considera que la proporción gastada en alimentos difiere entre hogares no sólo por el nivel de ingresos que ellos puedan tener, sino por diferencias en sus característica demográficas, en los precios relativos o en sus preferencias. Otros métodos surgidos en este mismo período han sido: el de las necesi dades básicas insatisfechas y los nutricionales o del consumo kilo calórico de sus miembros, más conocido como agregado de consumo (Feres, 2001).

Este enfoque surge en la segunda mitad de los años ochenta. Coincide con los primeros reconocimientos del fracaso de las políticas de ajuste, la teoría del chorreo. Se identifica con el enfoque $M$ ujeres en el Desarrollo (M ED), en el cual la desigualdad económica entre hombres y mujeres no está ligada a la subordinación sino a la pobreza. De esta manera se cambia el énfasis de la reducción de la desigualdad entre hombres y mujeres a la reducción de la desigualdad de ingresos.

Este enfoque no logra imponerse como el del bienestar aunque su instrumentación alcanza su expresión más clara en proyectos financiados por la cooperación internacional y agencias de desarrollo focalizado en las políticas sociales y familiares. El énfasis se atribuye a la perspectiva MED puesto por los planificadores del desarrollo y sus agencias en programas encaminados a que las mujeres puedan cubrir las necesi dades de su familia por medio de proyectos sól o para ellas, siendo las áreas en las que se han concentrado dichos proyectos: salud, planificación familiar, educación y capacitación para el trabajo y creación de pequeñas empresas.

La mujer, en este enfoque, es identificada como el sector de más pobreza, reconocien do su papel sustantivo en la satisfacción de las necesi dades de la reproducción de la fuerza de trabajo. Tampoco reconoce la esencia de la mujer en su papel de subordinación. Hace un reconocimiento de la falta de productividad en los hogares de bajos ingresos y vincula esa debilidad en la ausencia de acceso a la propiedad de la tierra, el capital y al mercado laboral. Y opera priorizando el trabajo para las mujeres en proyectos de generación de ingresos. Este enfoque ignora el rol reproductivo de las mujeres y sus responsabilidades en el hogar. A demás, asume que las mujeres tienen tiempo libre lo que incrementa el tiempo y tareas de su jornada de trabajo (Renzi, 2002).

\subsection{El enfoque de la eficiencia}

El enfoque de la eficiencia se ha desplazado de las mujeres ha- cia el desarrollo, bajo el supuesto de que una mayor participación económica de las mujeres del tercer mundo se vincula automáticamente a una mayor equidad. Coincide con la aplicación más intensa de los programas de ajuste estructural, dentro del contexto de la recesión mundial ocurrida en los años 80 . Esto ha permitido que organizaciones como el Banco M undial (BM), la O rganización para la C ooperación y el D esarrollo Económico (OECD) y la U nited States A gency for International D evel opment (U SA ID) propongan que un incremento en la participación económica de las mujeres en el desarrollo une la eficiencia con la equidad.

El enfoque de la eficiencia centra su atención en la capacidad de entrega de las mujeres a las exigencias del tiempo y trabajo que plantea la situación de ajuste. A rgumenta el esfuerzo de las mujeres, el sacrificio y su solidaridad para dar todo de sí a los demás. L os recortes en los gastos sociales son amortiguados por la elasticidad del trabajo de las mujeres en la autoproducción de alimentos, cambios en los hábitos de compra y patrones de consumo (Renzi, 2002).

Las críticas al enfoque se centran en que: el énfasis no está en las mujeres, sino en el crecimiento económico y focaliza a la mujer desde un punto de vista utilitario (R enż, 2002. También se considera que este enfoque sólo satisface necesidades prácticas de las mujeresa costa de más horas dedicadas al trabajo no remunerado, y limita el número y la calidad de la satisfacción de necesidades prácticas de género por la reducción de los recursos. A demás, el trabajo no remunerado de las mujeres y niños se amplía, como un mecanismo de funcionamiento eficiente del sistema económico, y por consiguiente, deja en mayores condiciones de vulnerabilidad a las mujeres.

\subsection{El enfoque del empoderamiento}

El enfoque del empoderamiento se originó a partir de la insatisfacción con el enfoque de la equidad de las M ujeres en el DesarroIlo (MED). U no de los supuestosfundamentales del enfoque de equidad que cuestiona el enfoque del empoderamiento es el relacionado con la interrelación entre poder y desarrollo implícito en los enfoques previos. $Y$, es que aún reconociendo la importancia de que las mujeres aumentan su poder, identifica éste menos en términos de dominación sobre otros y más en términos de la capacidad de las mujeres de incrementar su propia auto confianza en la vida e influir en la dirección del cambio, mediante la habilidad de ganar el control sobre recursos material es y no material es fun damental es ( $M$ oser, 1991: 110, citada por Riquert, 1998).

El enfoque del empoderamiento concibe a la mujer como capacitada y potenciada para dinamizar un cambio positivo en sus modos de vida habituales. Promueve valores como incrementar la autoestima, seguridad y capacidad de influir en la orientación de los cambios y no en la búsqueda de posiciones de dominio. Y, su fundamentación se centra en las relaciones de poder. A demás, plantea una visión más comprensiva, pues cuestiona el contexto social y económico para el logro de relaciones cualitativamente diferentes entre hombres y mujeres. Reconoce el triple rol de las mujeres y a 
partir de sus propias organizaciones trata de elevar la conciencia de las mujeres y hacerlas influyentes desde la base hasta las posiciones de más jerarquía en la sociedad.

El enfoque del empoderamiento supone un proceso progresivo con 5 niveles: i) Mejoramiento de las condiciones de vida. Es decir, el bienestar asociado a los aspectos materiales. ii) A cceso a recursos materiales y financieros necesarios para el desarrollo y la producción. iii) Concientización sobre la temática de género -subordinación, autonomía, autoestima y violencia de género-. iv) Participación de la mujer en la toma de decisiones a todos los niveles de la gestión del desarrollo. Por ejemplo, en la intervención efectiva en todas las fases de un proyecto. v) Control de su vida a partir de la articulación consecutiva y sinérgica que se da en las fases anteriores. Significa el paso de un nivel a otro y se concreta en la elevación y mejoramiento de las condiciones de vida de las mujeres.

Este enfoque de la pobreza toma en cuenta el enfoque de género. Evita el enfrentamiento con las estructuras subordinantes y emplea las necesi dades prácticas para construir apoyos seguros mediante la combinación de las actividades que satisfacen necesidades inmediatas, con formación directa o indirecta sobre los derechos, subordinación, autonomía, autoestima, violencia sexual y doméstica. $C$ abe señalar que el empoderamiento de las mujeres no supone elevar su status y su grado de dominación frente a los hombres, sino que supone una redistribución del poder.

En la aplicación de este enfoque se hace necesario tomar en cuenta aspectos tales como la opción de generar cambios desde las bases en la visión de los hombres lo cual supone procesos de muy largo plazo. El enfoque desafía el status quo de las relaciones de género y otras relaciones de iniquidad. Por cuanto su aplicación ha ganado adeptos a partir de perfeccionar su metodología y demostrar su eficacia. UNICEF ha asumido los grandes lineamientos del empoderamiento como enfoque oficial para el trabajo con mujeres.

\subsection{Hacia un nuevo enfoque de Pobreza}

El concepto de pobreza se está modificando día a día. Tradicionalmente la pobreza se suele reducir en algunos casos a la ausencia de bienes materiales 0 al precario ingreso y ha sido definida en otros, como la incapacidad que tienen las personas de vivir una vida adecuada (PNU D, 1997).

A ctualmente es concebida la pobreza como un fenómeno multidimensional y dinámico, en tanto es un hecho social e histórico. El concepto de pobreza ha sido relacionado a la ausencia de bienestar. Sin embargo se considera que el bienestar humano no puede medirse sólo a través de variables económicas porque ello esconde el problema de las relaciones entre las personas, los grupos socialesy las fuerzas de poder que condicionan la distribución no sólo de los ingreso, sino también de las oportunidades que tienen las mismas para gozar una vida digna (Renzi, 2002). De ahí, el surgimiento de nuevos enfoques surgidos en los últimos años.

\subsubsection{Enfoque de las capacidades}

El enfoque de las capacidades ha sido desarrollado principalmente por A martya Sen. Constituye otro punto de partida para el análisis de la pobreza y el bienestar de las personas. Enfatiza en el anál isis asociado al estándar de vida o calidad de vida y cuestiona el enfoque tradicional porque iguala los conceptos de "utilidad" y pobreza.

U no de los planteamientos de Sen ha sido considerar que los bienes no son los objetos, ni son éstos los que determinan el bienestar, pues ello no determina las actividades que una persona puede realizar, y las actividades en general están determinadas por las capacidades o impedimentos de cada persona. Considera que el nivel de vida de las personas puede determinarse por la comparación de los niveles de "utilidad" de las personas, pero, dado que la utilidad es una percepción mental subjetiva ante la ejecución de una capacidad, no puede utilizarse para evaluar con objetividad el nivel de vida de una persona $\mathrm{O}$ una sociedad. H a planteado que el enfoque de las capacidades puede ser complementario al análisis económico utilitarista, ya que el primero se presenta como un paso intermedio que conecta la utilidad con el consumo de bienes. Ravallion (1998).

A esta discusión se fue incorporando el enfoque "absoluto" y el enfoque "relativo". Según Spicker (1999) esa diferencia está relacionada principalmente con las interpretaciones de cada sociedad en relación a donde determina las necesidades. Bajo esta concepción las personas tienden a percibir su propio bienestar en función del bienestar de los demás y está muy condicionado al tipo de sociedad en el que cada persona vive. Esta discusión ha llevado al análisis de la desigualdad en la distribución del ingreso. Para Sen, pobreza y distribución son dos conceptos diferentes aunque fenómenos relacionados y analiza el ejemplo de que si se reduce considerablemente el ingreso de un país, y muchas personas dejan de tener recursos para alimentarse adecuadamente, este hecho no será considerado como un aumento de la pobreza por un enfoque puramente relativista, si la distribución del ingreso no ha cambiado. Por ello, Sen plantea que no basta analizar la pobreza como un simple problema de distribución del ingreso.

A Igunos autores, como Townsend, han estudiado la pobreza en función de la noción de "privación relativa". Parte de la idea que la pobreza depende de la riqueza general y, tomando en cuenta que ésta no es constante en el tiempo, concluye que el estándar para identificar a las personas que viven en situación de pobreza debe definirse en función del nivel general de ingresos. A sí la medición de la pobreza si empre tomará en cuenta los nuevos bienesy actividades necesarios para participar adecuadamente en la sociedad, a medida que crece el nivel general de vida. Otros impulsan el criterio "relativo" y plantean la ausencia en dicho enfoque de los crecientes requerimientos sociales. A martya Sen, por su parte señala que si bien la sociedad determina ciertas necesidades, no puede negarse la existencia de un "núcleo irreductible" de pobreza absoluta, indepen- 
dientemente del nivel de ingresos de algún grupo referencial. Ese núcleo esta determinado por las necesidades, cuya satisfacción representa sin lugar a dudas una situación de privación, como por ejemplo la inanición.

De esta manera, el enfoque de las capacidades determina que la pobreza es absoluta en el espacio de las capacidades y relativa en el espacio de los bienes. La falta de una capacidad es absoluta porque no depende de si otras personas la han satisfecho o no. Los aportes de Sen fueron recogidos por $\mathrm{M}$ ax $\mathrm{N}$ eef, diferenciando entre necesidad y satisfactores, y señalan do que las necesidades son absolutas ya que son las mismas en todas las culturas y todos los períodos históricos, pero los satisfactores de esas necesidades están determinados culturalmente y, por tanto, pueden ser distintos en diversas sociedades.

A ún cuando en dependencia del contexto económico, social y las con diciones particulares de cada país la pobreza adquiere dimensiones diferentes. Renzi señala que existen elementos comunes que originan las condiciones de insatisfacción de las personas, siendo éstas las si guientes: i) La incapacidad de las economías de generar las oportunidades de empleo en la cantidad y calidad necesarias que aseguren a las personas un nivel de ingreso adecuado; ii) La presencia de mecanismos de generación y distribución del ingreso con propensión a una alta concentración en grupos minoritarios de la población; iii) La incapacidad de las economías de generar el ahorro interno que permita la provisión de ciertos servicios básicos en la cantidad y calidad adecuadas para la población; y iv) que esos factores estructurales, han sido reforzados en los últimos años por las políticas económicas restrictivas que se han instrumentado en el marco de los programas de ajuste estructural.

\subsubsection{Enfoque de la exclusión social}

El interés por la pobreza en Europa ha ampliado la discusión, no solamente a los aspectos ya discutidos sobre las privaciones múltiples (bajosingresos, viviendas en malas condiciones, bajo acceso a servicios de salud y educación), dentro del marco de 'exclusión social', sino a la forma en que se dan estas privaciones. A partir de ello, se identifican como escenarios de exclusión los sistemas legales y democráticos, los mercados, la provisión de derechos, recursos y relaciones ${ }^{6}$ del estado benefactor, la familia y la comunidad (CEPA L, 2002). Ello hace suponer que ha surgido un nuevo elemento introducido al definir la pobreza, es la consideración de que la máxima expresión de la pobreza es la falta de participación en los procesos sociales o "exclusión social" (FLA C SO, 1996).

A I hablar de exclusión social, se agrega a la carencia material 0 falta de ingresos otro factor, relativo a las relaciones sociales. Con ello se dimensionan dos aspectos de la pobreza: la carencia material y la capacidad real de acceder o participar de las oportunidades básicas del sistema social, en términos de educación, salud, higiene ambiental, vivienda, agua, etc. y de participar del sistema político, en organizaciones de todo tipo y en la toma de decisiones.
Estudios realizados por diferentes autores relacionan la exclusión social con la escasez de oportunidades. A I respecto, se plantea que la exclusión social es una escasez crónica de oportunidades y de acceso a servicios básicos de calidad, a los mercados laborales y de crédito, a condiciones físicas y de infraestructura adecuada, y al sistema de justicia (BID:2001). Dicha exclusión se hace más fuerte para Ios individuos que pertenecen a múltiples grupos catalogados como excluidos como son los grupos étnicos y los discapacitados, entre otros.

\subsubsection{Enfoque de las 0 portunidades, seguridad y empoderamiento}

Otros conceptos nuevos asociados a la pobreza son: el de las oportunidades, la seguridad y el empoderamiento de ahí que se considere la asociación de ellos a un nuevo enfoque que explique la pobreza. En el Informe de Desarrollo M undial 2000-2001, dedicado a la lucha contra la pobreza, se propone una estrategia de lucha contra la pobreza sintetizada en los principios de oportunidad, empoderamiento y seguridad ( $R$ enzi, 2001). Ello podría indicar el surgimiento de un nuevo enfoque de la pobreza en el que se tomen en cuenta estas tres dimensiones: oportunidad, empoderamiento y seguridad.

La O portunidad se asocia al acceso a las opciones materiales como el acceso al mercado laboral, a créditos, propiedades, al desarrollo de infraestructura adecuada, a condiciones físicas, a la educación, a la salud y a un sistema de justicia imparcial en las dimensiones: Económica y Social. Por consiguiente, aunque el crecimiento económico sigue siendo clave para el desarrollo económico, cada vez adquiere más importancia la calidad de dicho crecimiento y ello está asociado a las oportunidades. Las opciones deben alcanzar a las poblaciones pobres y para ello se requiere de instituciones nacionales y locales que lo operativicen (Renzi, 2001).

El concepto de empoderamiento esta claramente arraigado a la noción de poder y en su contraria, la impotencia o ausencia de poder, tanto de los pobres, las mujeres y los grupos étnicos. La impotencia in dica una ausencia total de poder. (Kabeer N aila, 1988: 236). Es también definido el empoderamiento como la expansión de la libertad de escoger y de actuar, por cuanto significa aumentar la autoridad y el poder del individuo sobre los recursos que afectan su vida. $^{7}$

En el estudio "La economía, el bienestar y el mercado laboral en N icaragua", Renzi (2002) señala que el Empoderamiento está asociado a la sel ección y aplicación de medidas públicas que respondan a las necesidades de las comunidades y depende de los procesos políticos, social es e institucionales en una continua interacción. Ello significa, que la conformación de estructuras institucional es estatales y sociales debe facilitar el acceso de las personas que viven en situación de pobreza a los mercados y a aquellas oportunidades que les permita potenciar sus capacidades. Por lo que se requiere de la existencia de procesos que incluyan la rendición de cuentasy la transparencia en la gestión pública. 
Los grupos relegados de la sociedad que a través de la historia han buscado empoderarse en defensa de sus derechos son los grupos étnicos, los pobres y lasmujeres. Estas últimas lo han hecho a través de las organizaciones feministas, cuyo objetivo se centra en la igualdad formal de las mujeres con respecto a los hombres. Por consiguiente, en la medición de la pobreza, el empoderamiento es clave ya que es el único componente que alude a lo subjetivo, a lo identitatario.

La Seguridad consiste en la cobertura de las N ecesidades Básicas para una vida digna en las dimensiones Sociales y Economicas. Se refiere (Renzi, 2001) a la reducción de la vulnerabilidad derivada por crisis económicas, políticas, enfermedades, discapacidad y violencia personal y por desastres naturales.

Para garantizar la seguridad de las personas se requiere de acciones de políticas y programas que permitan a la población enfrentar en mejores condiciones los factores de riesgo. Para esto, el incremento de activos en manos de la población que enfrenta una situación de pobreza es clave, así como la capacidad para la autogestión y los mecanismos de protección con una activa participación de las comunidades afectadas por la inseguridad ( Renzi, 2001) . C abe señalar que en este enfoque, los tres aspectos oportunidad, empoderamiento y seguridad son complementarios mutuamente, ya que tienen incidencia en las causas de la pobreza.

\section{Enfoque de Vulnerabilidad Social}

U no de los nuevos enfoques teóricos relacionados a pobreza surgidos en losúltimos años es el de vulnerabilidad social. La noción del vulnerabilidad es entendida como un proceso multidimensional que confluye en el riesgo o probabilidad del individuo, hogar o comunidad de ser herido, lesionado o dañado ante cambios o permanencia de situaciones externas y/ o internas (Busso, 2001). También es entendida como una condición social del riesgo, de dificultad, que inhabilita e invalida, de manera inmediata o en el futuro, a los grupos afectados, en la satisfacción de su bienestar-en tanto subsistencia y calidad de vida- en contextos socio históricos y culturalmente determinados. ${ }^{8}$

El concepto de la vulnerabilidad está asociado a la situación de las personas, hogares o comunidades derivada de un conjunto de elementos que influyen en sus vidas y que se reflejan en estados anímicos y de carácter subjetivo. ${ }^{9}$ Tales como: i) La fragilidad o indefensión originada por los cambios en el entorno externo derivado de las grandes tran sformaciones en el ámbito mundial; ii) el desamparo institucional derivado del nuevo papel desempeñado por el Estado; iii) la debilidad de las personas y sus hogares para aprovechar las oportunidades que se les presentan, y iv) la inseguridad que paraliza, desmotiva para pensar estrategias.

El enfoque de Vulnerabilidad Social permite poner en el centro de la discusión la articulación de aspectos como: Ias dotaciones inicial es de activos y las formas de su reproducción; el uso de estrate- gias diferenciadas por parte de las personas, hogares y comunidad en diferentes dimensiones; y la conformación de escen arios socioeconómicos y políticos con responsabilidades compartidas (gobiernos locales, nacionales, la sociedad y las diversas instancias internacionales). Y, remite al análisis de la relación dialéctica entre entorno y el "interno" que presenta determinadas características que califican la unidad de análisis como vulnerable en función de los riesgos a los que están expuestos y de las capacidades de respuestas ante eventuales choques adversos. Combinándose esta exposición a riesgos con las características internas básicas de las personas, hogares, grupos o comunidades que enfrentan (a la vez que generan) cambios en su contexto de referencia (Renzi, 2002).

El enfoque de la vulnerabilidad social está estrechamente relacionado al enfoque de las oportunidades, entendién dose por un conjunto de oportunidades ( Renzi, 2002) , a las posi bilidades de acceso a los mercados de bienes y servicios para realizar intercambios y transacciones, a la posibilidad de acceder a medios de subsistencia, empleo, protección social y a derechos de ciudadanía que permitan a las personas, hogares y comunidades alcanzar un nivel de bienestar a través del tiempo.

El concepto de vulnerabilidad centra su atención en la capacidad habilitadora de la responsabilidad compartida entre personas, hogares e instituciones respecto a las condiciones y resultados de las políticas públicas. Si bien la responsabilidad compartida no exime de las responsabilidades al Estado en sus diferentes niveles territoriales, la idea de activos permite distinguir en la formulación de políticas, los motivos, los condicionantes, las actitudes y los resultados de las estrategias de vida de individuos y hogares. ${ }^{10}$

C abe señalar que el concepto de "activo" no se refiere sólo a los ingresos, aunque estos por diversas vías permiten obtener ingresos, entre otras cosas. Pueden ser físicos, financieros, sociales, humanos y ambientales. Los activos, condicionan también la capacidad de respuesta que tien en las personas, los hogares y las comunidades a los riesgos tanto de origen natural, como social, económico y político (Renzi, 2002).

A ctivos físicos son los medios de vida como la vivienda, animales, recursos naturales, bienes durables para el hogar y el transporte familiar, etc., usados para mantener y reproducir la vida en el hogar; también incluyen los medios de producción, como los bienes que se usan para obtener ingresos o intercambio de bienes (herramientas, maquinarias, transporte para uso comercial, etc.). Los activos financieros son el ahorro monetario, créditos disponibles (cuenta corriente de bancos, tarjetas de crédito, fiados de almacenes, etc.), acciones, bonos y otros instrumentos financieros de uso habitual en el sistema financiero formal einformal. Los activos humanos son los recursos de que disponen los hogares en términos de cantidad y calidad de la fuerza de trabajo del hogar, y el val or agregado en inversiones en educación y salud para sus miembros; mientras que los activos sociales, conocidos como capital social son intangibles y se refieren a las relaciones sociales. Son una forma y un atri- 
buto colectivo o comunitario que incluyen vinculaciones solidarias, lazos de confianza y relaciones de reciprocidad articuladas en redes interpersonales. Como activos ambientales se consideran a las características y atributos del ecosistema y la biosfera que se relaciona con el nivel de bienestar, la calidad de vida y la sustentabilidad actual que tiene una sociedad a partir de sus procesos de reproducción de individuos, hogares y comunidades en un territorio específico (Renzi, 2002).

\section{Vulnerabilidad Sociodemográfica}

La noción de vulnerabilidad demográfica surge a partir de la aparición de temas derivados de los cambios en los model os económicos como son: el ritmo, crecimiento y composición de la población, la distribución espacial, las migraciones, la fecundidad adolescente, la salud reproductiva, la calidad de la esperanza de vida y los derechos de ciudadanía, entre otros. Este enfoque considera que hay variables demográficas que constituyen una situación de desventaja adicional para las personas, hogares y grupos de población como lo son: la fecundidad alta y temprana, el evadosíndices de dependencia demográfica en el hogar, la estructura de la población a partir del sexo y edad, la jefatura de hogar -muy joven o muy anciana- y una creciente participación de jefatura femenina, inadecuados cuidados de la salud y la localización residencial en áreas riesgosas (Renzi, 2002). Por consiguiente, el enfoque de la vulnerabilidad socio demográfica, facilita analizar escenarios a más largo plazo con cierto grado de confiabilidad y, de esa manera, formular políticas que apuntan a incidir sobre las verdaderas causas que ponen a las personas, hogares y comunidades en condiciones de vulnerabilidad.

A partir de los diferentes enfoques de la pobreza antes expuestos, Ilegamos a considerar que el concepto de vulnerabilidad sociodemográfica incorporándole el enfoque de género esel que mejor explica la pobreza. Es decir, que a los el ementos considerados en el enfoque de la vulnerabilidad sociodemográfica se debe agregar el elemento del empoderamiento. A continuación se presenta una revisión rápida sobre las diferentes formas de medición de la pobreza puestas en práctica en los últimos años.

\section{FORMAS DE MEDICIÓN DE LA POBREZA}

Desde el punto de vista metodológico el proceso de medición de la pobreza abarca cinco elementos: i) La definición de pobreza; ii) los supuestos sobre los datos utilizados referidos al universo cubierto y la unidad de análisis; iii) el método empleado para la medición y los indicadores empleados con un enfoque conceptual determinado; iv) Ia elaboración de una o varias dimensiones de pobreza; v) y el punto de cortes entre pobres y no pobres.

Por consiguiente, a la par de la introducción de nuevos aspectos en los debates sobre el concepto de pobreza, se ha desarrollado toda una discusión sobre cómo medirla. El deseo de aprehen der mejor la real idad cedió paso a la necesidad de encontrar formas factibles de medir la pobreza. U no de los problemas que se enfrentan es que se mide la pobreza a partir de una dimensión, cuando se sabe que es un fenómeno multidimensional. Otro de los problemas se relaciona con los indicadores y es que aún con los indicadores más incluyentes y participativos no es posible hacer agregaciones o generalizaciones con ellos o utilizarlos para producir cifras comparables a nivel nacional e internacional. En real idad, Ios debates surgen de las diferencias epistemológicas y de la consideración predominante de que la 'objetividad' y los métodos cuantitativos son más aceptables y científicos que los cualitativos. (H arraway; 1998).

A I gunos supuestos utilizados para medir la pobreza son en función de la temporalidad y del método, lo que hace ver a la pobreza a la hora de medirla como sincrónica o diacrónica, como causal o como factorial.

1 La pobreza es sincrónica o diacrónica / causal o factorial

El tipo de medición adoptado depende de una distinción entre pobreza como concepto sincrónico, que sucede al mismo tiempo o como concepto diacrónico, que no sucede al mismo tiempo. Los sincrónicos buscan como analizar una causalidad que se extiende en el tiempo, es decir que hacen uso de las tablas de contingencia o tablas cruzadas y de la comprobación de hipótesis correlacionadas a través del $\mathrm{C}$ hi cuadrado. Hacen uso de datos censales o de datos obtenidos a través de encuestas de hogares (EH ). Dichos datos se conocen como datos "cross-sectional". Por su parte, los diacrónicos utilizan como medidas estadísticas, las series de tiempo.

El tipo de medición también depende del enfoque explicativo que se tiene, y este puede ser: causal o factorial. El causal, únicamente aplica un análisis bivariado a través del cruce de dos variables y trata de comprobar hipótesis correlacionadas. Esto lo realizan a través del $\mathrm{C}$ hi cuadrado o del coeficiente de regresión. Ejemplo de este tipo de estudios, es en el que tratan de explicar la desnutrición infantil con variables socioeconómicas (vivienda, escolaridad, clase social). Mientras que el factorial, busca como encontrar la interrelación entre varias variables, es decir que logra un análisis de mayor alcance, aplicando el análisis multivariado para la búsqueda de un factorial que explique la correlación entre las diversas modalidades de las variables. A unque hay quienes miden la pobreza a partir de una sola variable: el ingreso, lo cual es totalmente deficiente ya que la pobreza es multidimensional. En este caso, la pobreza es un fenómeno inobservable como tal, sino que se le destaca a través de una red de variables que se interrelacionan. Es decir que la pobreza es el resultado de la interrelación de varias variables: desnutrición, desempleo, mortalidad, analfabetismo, etc.

$\mathrm{H}$ ay quien es se han propuesto analizar factorialmente otros elementos, como la necesidad de pensar en lo que sucede con las personas al interior del hogar más que seguir considerando el hogar como la unidad básica de análisis, aunque no han sido tomados en cuenta 
por razones que podemos considerar más ideológicas que metodológicas. Sin embargo, los estudiosy mediciones generalmente realizados sobre pobreza, permiten solamente dar cuenta de la población total y por sexo y por consiguiente de la población femenina. M ientras que la pobreza relativa de las mujeres por razones de género no se aborda debido a la no incorporación de categorías de anál isis que den cuenta de los factores estructurales o determinantes de la situación de la mujer. (CEPA L; 1997).

$\mathrm{H}$ ay métodos de medición diferentes y por ende, diferentes indicadores para medir la pobreza. A continuación se presentan los principales $M$ étodos de medición de la pobreza puestos en práctica en Latinoamérica. Los más utilizados son: el N BI o de N ecesidades Básicas Insatisfechas; el LP o de Línea de Pobreza, el de Consumo calórico y el DIM o de Distancia, intensidad y magnitud.

\section{M étodo de Línea de Pobreza (LP)}

El método de línea de pobreza hace énfasis en el ingreso o consumo de la población y establece los límites de la pobreza relativa y absoluta, a través de distintas mediciones.

U na forma de abordar el método de línea de pobreza es a través de los ingresos de las personas de los hogares. La fuente principal de medición es la sumatoria de todos los ingresos de las personas de los hogares (monetarios y no monetarios) que se relacionan a diferentes conceptos: ingreso monetario por trabajo, salarios en especie, autoconsumo, subsidios, transferencias, remesas, etc. La sumatoria de dichos ingresos se relaciona al costo de una canasta básica alimenticia para definir el límite de la extrema pobreza o indigencia. Por lo general, ese límite se define a partir de registros históricos, pues se asume que es el mínimo necesario para que las personas tengan un nivel de vida decente.

La línea de la pobreza general, se define en el valor de ese mínimo multiplicado por dos. Ese factor se sustenta en los diferentes estudios realizados para la región que muestran que esa es la relación entre un mínimo de subsistencia y el costo de otros bienes y servicios que permite la reproducción social. Por lo general, esta metodología se confronta con distintos puntos de vista sobre el contenido de lo que se denomina canasta básica. En al gunos países para superar esa discusión se toma como referencia el salario mínimo legal. En consecuencia, para la utilización del método de la línea de pobreza no hay reglas fijas y depende de las definiciones propias de cada país.

Bajo el método de la línea de pobreza por ingresos, se considera pobres a las personas que viven en hogares con ingresos menores al costo de una canasta básica de bienes y servicios, considerada como mínima para satisfacer las necesidades de subsistencia de todos sus miembros. Son hogares en situación de extrema pobreza aquellos cuyos ingresos son iguales o inferior al costo de una canasta básica alimenticia o el salario mínimo, según sea el caso. Los que tienen ingresos equival entes a dos canastas básicas alimenticias o menos 0 dos salarios mínimos, según sea el caso, se consideran hogares o personas en situación de pobreza, y no pobres aquellos cuyos ingresos son superior al equivalente de las dos canastas básicas o superiores al límite fijado para la medición de la pobreza.

Dadas las dificultades de establecer ese valor monetario mínimo de referencia para medir la pobreza, algunos estudios sobre la pobreza en $\mathrm{N}$ icaragua han cuantificado la pobreza a partir de los ingresos por hogar y per capita pero relacionados al nivel de las líneas de pobreza definidas para el calculo del agregado del consumo.

Este método presenta ventajas y desventajas. Por lo general, se argumenta que la declaración de ingresos presenta subregistro, derivándose de ello posiblemente un nivel de pobreza mayor y presenta mayores fluctuaciones en el corto plazo. A Igunas de las críticas que se hace a los indicadores de Línea de Pobreza es que no sólo aprehenden la natural eza de forma dinámica y multidimensional a partir de la privación y el bienestar, sino que invisibilizan las diferencias, tanto entre comunidades y entre hogares dentro de una comunidad, como las diferencias entre los hombres y las mujeres y entre las mujeres.

Tomando en cuenta el enfoque de capacidades, no siempre el indicador o línea de pobreza establecido para la medición, es aceptado por la población, debido principalmente a que en su calidad de vida incluyen otros aspectos como por ejemplo buenas condiciones de trabajo, autodeterminación, acceso al poder, entre otros, elementos que no entran en la contabilización de los métodos tradicionalmente utilizados. Sin embargo, a pesar de las críticas hacia el enfoque de "pobreza-ingreso y el desarrollo de enfoques e indicadores alternativos, los indicadores de línea de pobreza han sido empleados por el G obierno de N icaragua.

\section{Método de Agregado de Consumo}

Este método mide la capacidad económica de las familias para al canzar niveles mínimos de consumo. Cuantifica el gasto derivado del consumo de las personas del hogar y los convierte a un mínimo de requerimientos calóricos diarios por adulto equivalente a 2.226 kcal. El Banco M undial ha realizado la cuantificación del gasto derivado del consumo de las personas del hogar y los convierte a un mínimo de requerimientos calóricos diarios por adulto por considerarlo más confiable. En términos económicos esto se valora en US \$ 237 dlls por año por persona, lo que equivale a 0.60 dlls por persona por día.

Sobre la base de esa línea de consumo, se establece la definición de pobreza extrema para aquellos hogares o personas cuyo consumo se ubica por debajo de ese nivel de consumo mínimo. Para el procesamiento de los resultados de lasEncuesta de medición de $\mathrm{N}$ ivel de vida de 1998 y 2001 en N icaragua (EM N V 98 y EM N V 01), ese límite fue fijado en C \$2,489 o U S\$237 y C \$2,691 o U S\$ 202 por persona para losañosen 1998 y 2001 respectivamente. C onstituyen hogares o personas no pobres, aquellas cuyo consumo supera este último valor. Se consideró como hogares pobresa los que consumen el mínimo calórico más un porcentaje adicional para cubrir otros consumos básicos como vivienda, salud, educación, y vestuario. 
El método del agregado de consumo es más complejo en su cálculo pues requiere de ajustes de precios. A I gunos argumentan que la declaración del consumo refleja mejor la situación de mediano plazo de los hogares o personas. Es decir, no sufre de variaciones significativas en el corto plazo. Sin embargo, es forma de medir la pobreza, también ha sido cuestionada por no tomar en cuenta el enfoque de género. A I respecto B rashaw señala que dentro de los hogares los hombres consumen de manera desproporcionada una cantidad mayor de los recursos del hogar, tanto en el consumo de alimentos como por los ingresos que ellos retienen para adquirir cosas para su propio consumo ( 'juegos de azar, beber con los amigos o andar con otras mujeres).

\section{M étodo de Necesidades Básicas Insatisfechas (NBI)}

El M étodo conocido como de N ecesidades Básicas Insatisfechas ( $\mathrm{N} \mathrm{BI}$ ) considera como pobre a todos aquellos que no cumplen por lo menos una necesidad básica, y considera que son hogares no pobres aquellos en los que no se presenta insatisfacción en ninguno de los indicadores que componen el índice agregado de las necesidades básicas insatiffechas.

La CEPA L ha optado por los siguientes indicadores: A cceso a viviendas que aseguren condiciones mínimas de habitabilidad para el hogar, integrado por las variables: tipo de construcción, materiales de los pisos y paredes exteriores y hacinamientos. Acceso a servicios básicos que aseguren un nivel sanitario adecuado, integrado por las variables: abastecimiento de agua en la vivienda, disponibilidad de servicios higiénicosy sistema de eliminación de excretas. Capacidad económica para alcanzar niveles mínimos de consumo, integrado por las variables: probabilidad de insuficiencia de ingresos entendida como hogares con jefes de hogar menor de 45 años de edad y menos de 5 años de instrucción primaria y con 4 personas por ocupado, o para las áreas rurales, hogares con jefes de hogar mayor de 45 años de edad y menos de 2 años de instrucción primaria y que tienen cuatro o más miembros por ocupado (CEPA L, 1989).

Sin embargo, en Nicaragua para la aplicación del método se toman en cuenta 5 indicadores de carácter estructural, los cuales son: hacinamiento, servicios insuficientes, vivienda inadecuada, dependencia económica y baja educación. A partir de ellos se construye un indicador compuesto con el cual se califica a los hogareso a sus habitantes en determinados niveles de pobreza. Se considera que son hogares no pobres, aquellos en los que no se presenta in satisfacción en ninguno de los indicadores que componen el índice agregado de $\mathrm{N} \mathrm{BI}$.

A cada uno de losindicadores seleccionados, se les aplican criterios de insatisfacción sobre la base de referencias internacionales, los que han sido ajustados con base a las características de $\mathrm{N}$ icaragua. El hacinamiento por ejemplo: toma en cuenta el número de personas en el hogar y el número de cuartos para dormir de la vivienda. Se considera inadecuado cuando el número de personas por cuarto es de cuatro o más en el sector urbano y de 5 o más en el sector rural.
Servicios Insuficientes, se refiere al acceso de agua potable adecuada y al servicio sanitario. En el caso de lasviviendas urbanas, el nivel de insatisfacción se define si no hay inodoro o letrina y no se tuviera acceso al agua potable por la red pública. En el sector rural, la insatisfacción está dada por la carencia de servicios sanitarios y si el agua se extrae de río, manantial u ojo de agua, donde no se puede hacer control de calidad. Vivienda inadecuada es una combinación del estado y tipo de materiales de la pared, techo y piso. Se considera adecuada la vivienda si por lo menos dos de esas variables tuvieran condiciones aceptables. Vivienda inadecuada es la presencia de un material aceptable o ninguno. Dependencia económica combina el nivel educativo de la jefatura del hogar y la tasa de dependencia, definida como el número de personas que dependen de una persona del hogar que esté con algún empleo. La dependencia económica se define cuando hay dos o más personas por cada ocupado y si además el o la jefa de hogar tiene una educación de primaria incompleta o no tiene educación. Para el sector rural, la tasa de dependencia se define en tres o más personas por ocupado. Y, Baja educación mide el acceso a los servicios de educación básica de los y las niñas entre los 7 y 14 años. La baja educación se define cuando hay presencia de niños de esas edades que no asisten a la escuela (Renzi, 2002).

Bajo esta metodología, la pobreza se define a partir de la presencia de uno o más indicadores insatisfechos y son extremadamente pobres aquellos que presentan dos o más indicadores no satisfechos.

El M étodo de Necesidades Básicas Insatisfechas al igual que los otros métodos, tiene el inconveniente de sintetizar en un solo indicador diversas necesidades y el grado en que éstas son satisfechas por lo que también ha sido cuestionado.

5 Método Distancia, intensidad y magnitud de la pobreza o método (DIM)

Este M étodo es conocido como de Distancia, intensidad y magnitud de la pobreza. $C$ al cula la pobreza sobre la base de una distancia entre el hogar observado y el hogar ideal. Se mide a través de fórmulas matemáticas y de la aplicación del análisis factorial. Busca como construir factores de pobreza a través de un conjunto de indicadores. Todos los hogares medidos tienen un valor que mide su grado de pobreza que va desde muy pronunciada, hasta ninguna pobreza. En algunos casos aplican una técnica de regresión y seleccionan indicadores que explican una consecuencia última de la pobreza que es la mortalidad infantil.

Constituye un aporte interesante al método del NBI y del LP. Busca corregir algunos defectos del N BI. En primer lugar, busca una técnica de selección de los indicadores que no estén priorizados. En segundo lugar, busca una solución al problema del peso de los indicadores parciales en IA P (Indice A gregado de Pobreza). En tercer lugar, elabora una nueva manera de medir la dimensión de pobreza. En cuarto lugar, elabora un método de corte que no esté tan 
fuerte que lo adoptado por el NBI. Busca un punto de corte que combina el NBI y el LP.

La metodología adoptada es del tipo ex post. Se plantea que los indicadores de pobreza son los que más den cuenta de la consecuencia última de la pobreza que es la mortalidad infantil. Por lo tanto, dado ese supuesto, se seleccionan los indicadores en función de su capacidad explicadora de la mortalidad infantil, utilizando una técnica de regresión múltiple lineal. El procedimiento es el siguiente: se elabora una base muy amplia de variables posiblemente explicativas. $\mathrm{N}$ o importa el número. Se seleccionan unos 43 indica-dores de la Encuesta de Hogares, que abarcan todas Ias temáticas: salud, vivienda, empleo, demografía. Este método ha sido considerado como el mejor de los métodos por sus muchas ventajas y por ser más realista.

\section{El Método combinado}

Por las características de los métodos de medición arriba detallados, ni el método de NBI ni el de la Línea de Pobreza pueden analizarse aisladamente para extraer concl usiones so bre las con di ciones reales de la población. Como un intento de aproximar más el conocimiento de la pobreza, se ha definido el método combinado.

Por consiguiente, este método surge de la combinación de los resultados de la pobreza por LP y N BI. Este método permite clasificar a la población o sus hogares en cuatro tipologías: Integrados, en pobreza reciente, en pobreza inercial y en pobreza crónica.

La ventaja del método combinado es que permite disponer de una visión más realista del problema. Los métodos de ingreso y el de NBI por separado dan indicaciones sobre las consecuencias que determinadas políticas tienen o podrían tener sobre la población. Por tanto, el análisis individual de cada uno de ellos pueda dar una visión sesgada de la realidad. Los ingresos son sensibles a las variaciones coyunturales de la economía y del mercado laboral, por tanto, no permiten discernir si la insuficiencia de recursos que experimentan los hogares, es de carácter permanente o transitorio.

De igual manera, buenas o malas condiciones de al guno de los indicadores de N BI, tampoco da pautas sobre la extensión del tiempo en que se ha producido el deterioro y si depende de sus propias posibilidades transformar esa situación o ésta más bien obedece a las políticas gubernamentales.11 Por consecuencia, el monitoreo periódico del método combinado, permite dar respuesta a esos grandes interrogantes y de esa manera facilitar la orientación de políticas y acciones que apunten a reducir las brechas sociales, las que constituyen las bases de la ingobernabilidad. Por otra parte, el monitoreo de la evolución de la pobreza bajo este método, a través del tiempo y con metodología panel ${ }^{12}$ permite observar las ten dencias de movilidad o descenso social que se da en los hogares o personas.

7. Índices de Naciones Unidas

$\mathrm{N}$ aciones $\mathrm{U}$ nidas utiliza cuatro índices para medir: el desarroIlo humano, la pobreza y el género.

\section{Indice de Pobreza H umana (IPH)}

El Indice de Pobreza mide el cúmulo de privaciones que persisten en los seres humanos en las mismas dimensiones del Indice de D esarrollo H umano. Estas dimensiones son: la supervivencia, medida a partir de la probabilidad al nacer de no sobrevivir hasta la edad de 40 años; Ios conocimientos, a partir de la tasa de analfabetismo de adultos; y el aprovisionamiento económico general, tanto público como privado, reflejado por el porcentaje de personas que no utilizan fuentes de agua mejorada y el porcentaje de niños menores de 5 años que tienen un peso inferior al normal. También incorpora, en al gun os países otras dimensiones, como son la exclusión social a partir de los in dicadores si guientes: probabilidad al nacer de no sobrevivir hasta los 60 años de edad, la tasa de analfabetismo funcional de adultos, el porcentaje de la población que viva por debajo de la línea de pobreza de ingreso (con ingresos familiares disponibles inferior al $50 \%$ de la mediana) y la tasa de desempleo a largo plazo ( 12 meses a más).

\section{Índice de Desarrollo Relativo al Género (IDG)}

Este índice mide el progreso de las mismas dimensiones y utiliza los mismos indicadores que el IDH, pero refleja las desigual dades en el progreso entre el hombre y la mujer. Es el índice de Desarrollo H umano ajustado para determinar la desigualdad de género. A sí, mientras mayor sea la disparidad de género en el Desarrollo H umano básico, más bajo será el Índice de Desarrollo relativo al G énero (IDG ) de un país respecto al Índice de Desarrollo H umano (IDH ).

\section{Índice de Potenciación de Género (IPG)}

EI Indice de Potenciación de G énero revela si la mujer puede participar activamente en la vida económica y política. Se centra en la participación y la adopción de decisiones económicas y políticas. Registra los porcentajes de mujeres en el parlamento, entre los legisladores, Ios funcionarios superiores y administradores, los profesional es y los trabajadores técnicos, así como las di sparidades de género en el ingreso percibido, como reflejo de la independencia económica.

A diferencia del Indice de D esarrollo relativo al G énero, pone de manifiesto la desigual dad de oportunidades en esferas sel eccionadas. (Informe sobre el Desarrollo Humano, 2001)

10. Niveles de Análisis e indicadores utilizados en el análisis de la pobreza

La pobreza ha sido analizada a través de cuatro niveles de análisis que se relacionan con los indicadores, que son: de resultados, de satisfacción, de acceso y de consecuencias últimas.

El primer nivel de análisis de la pobreza abarca lo que se llama los indicadores de resultados que son la satisfacción de las necesidades dichas básicas. En general se contemplan los siguientes indicadores: alimentación, vivienda, salud, educación. Estos indicadores miden el grado de satisfacción de necesi dades básicas. El 
enfoque directo de medición de la pobreza se ubica en el nivel de resultados

El segundo nivel está compuesto de los medios de satisfacción, es decir de aquellos insumos que permiten al hogar satisfacer sus necesidades. Estos medios son: a través del mercado laboral, el ingreso del trabajo, los servicios gratuitos o subvencionados por el estado como es el agua, la luz, el alcantarillado, la educación, la vivienda y la salud, la capacidad de auto consumo del hogar, los seguros que defienden el hogar frente a los riesgos como pueden ser el IN SS, seguros particulares, solidaridad familiar y barrial. Y, constituyen la base de la medición de la pobreza según el método indirecto. Y, privilegia la variable ingreso, es decir que mide la pobreza al nivel de recursos.

El nivel de acceso agrupa aquellos indicadores que determinan el acceso a los medios de satisfacción o que, más bien, que interactúan hacia la satisfacción de las necesidades básicas.

M ientras que el de las consecuencias suele distinguir las consecuencias últimas de la pobreza como son la mortalidad y la inserción social deficiente. Se asemeja un poco al nivel de resultados.

\section{CONCLUSIÓN}

A manera de conclusión podría decirse que dado que históricamente la pobreza ha sido analizada con un enfoque globalizante que invisibiliza la situación de las mujeres, se hace necesario incorporar el enfoque de género en este análisis. Y por consiguiente, se hace necesario analizar las causas específicas de la pobreza de las mujeres, ya que aún a las mujeres que cuentan con ingresos se le hace más difícil poder transformarlos en capacidad de tomar decisiones o para poder elegir en qué utilizarlos. De ahí la importancia en considerar que las percepciones sobre el valor y la contribución al hogar, Ias normas sociales y los niveles de autonomía tienen impacto en la capacidad de las mujeres para tener voz en el proceso de toma de decisiones a todos los niveles.

A demás, cuando las mujeres sí toman decisiones, no mejoran su propio bienestar sino generalmente deciden en función de aumentar el bienestar general de la 'familia', lo cual se debe no solamente por las responsabilidades 'tradicional es' de cuido dela/osniña/ os, sino debido a que la construcción de la identidad femenina implica ser altruista (Branshaw, 2001).
O tra de las conclusiones es que el análisis de los determinantes de la pobreza tal a como lo plantea Brashaw requiere no solo de la profundización del conocimiento de losfactores de género, las variables de vinculación sectorial, la dependencia económica sino también de la vulnerabilidad social y demográfica. Ello a partir de que la vulnerabilidad de ciertos sectores social es se expone a mayores situaciones de riesgo profundizando así su pobreza. Por consiguiente, que un nuevo enfoque de la pobreza debería considerar elementos del enfoque de la vulnerabilidad sociodemográfica, pero con el enfoque de género. Siendo que, las causas de la pobreza de las mujeres operan a diferentes niveles o existen en diferentes 'sitios' de pobreza -a nivel de la sociedad en general debido a una discriminación institucionalizada, incluyendo los mercados de trabajo y el poder político; a nivel de la comunidad donde las normas sociales tienen impacto sobre los roles y respon sabilidades de hombres y mujeres y a nivel del hogar donde las relaciones desiguales de poder operan según sexo y edad, se hace necesario proponer nuevas dimensiones y nuevos indicadores para medir y analizar la pobreza.

$\mathrm{A}$ I respecto, en al gunos estudios real izados en $\mathrm{N}$ icaragua se ha dicho que las limitaciones que enfrentan las mujeres en el acceso a recursos es lo que impide romper con el círculo de la pobreza. Y, que el acceso a recursos como tierra, capital, vivienda, facilidades crediticias, empleo, tecnologías, insumos y extensión de servicios, son también considerados como cruciales para la producción agropecuaria y para dinamizar el resto de las actividades económicas sobre las que descansa la sobrevivencia de miles de nicaragüenses hombres y mujeres (Brashaw, 2001).

De ahí que se considere importante tomar en cuenta en los estudios de la pobreza tres elementos ya planteados por Branshaw (2001): que las mujeres tienen menores posibilidades para traducir su trabajo en ingresos- debido a su responsabilidad exclusiva del trabajo reproductivo, a la conceptualización de sus actividades productivas como una 'ayuda' a los hombres y a su concentración en el sector informal.

Es también importante señalar que una propuesta de dimensiones e indicadores para medir la pobreza debe basarse en un enfoque integrador y estructural que visibilice la situación de las mujeres en la sociedad a todos los niveles: macro, meso y micro. 